\title{
Laboratory Millimeter Wave Spectroscopy of Small Reactive Species
}

\author{
C. Demuynck, M. Bogey, H. Bolvin, M. Cordonnier, J.L. Destombes, A. Walters \\ Laboratoire de Spectroscopie Hertzienne, Universite de Lille, F-59655 Villeneu ve d'ascq \\ Cedex,France.
}

\section{Introduction}

The discovery of a large variety of molecules by radio astronomy has been a very strong motivation for the development of laboratory millimeter wave spectroscopy. Among them, the reactive species, neutral and/or ionic, have been early recognized as playing a very important role in the chemistry of the interstellar and circumstellar medium. While the laboratory spectroscopy of free radicals started relatively early, with the observation of the $\mathrm{OH}$ radical by the group of Townes (Dousmanis et al. 1955), the detection of molecular ions proved to be a much more difficult task, and the first millimeter line due to an ion was actually detected by radio astronomy (Buhl \& Snyder 1970). It was called "U89.2" until it was tentatively attributed to $\mathrm{HCO}^{+}$by Klemperer $(1970)$ on the basis of both considerations on the chemistry of the interstellar medium, and of ab initio calculations for the prediction of the expected line frequency. This identification was later confirmed by more elaborated ab initio calculations (Wahlgren et al. 1973, Kraemers \& Diercksen 1976), and by the observation of a transition attributed to $\mathrm{H}^{13} \mathrm{CO}^{+}$(Snyder et al. 1976), but the definite confirmation was the observation of the same transition in a laboratory glow discharge by the group of Woods (Woods et al. 1975).

Due to their key-role in the astro-chemistry (see for example Viala 1986 and references therein), molecular ions are of course of special interest to laboratory spectroscopists, but many other types of molecules are also very important: free radicals and reactive species are relatively abundant in the interstellar medium; small cyclic molecules have been searched for since the discovery of $\mathrm{C}_{3} \mathrm{H}_{2}$ in a wide variety of sources; refractory molecules have been detected in circumstellar envelopes, and the observation of rare isotopic forms can give information on the mechanisms of isotopic fractionation. In all cases, the detection of these species request a preliminary observation of their spectra in the laboratory.

In addition to its significance for astrophysics, millimeter wave spectroscopy is a very powerful tool for probing the physico-chemistry of low pressure flames and 
plasmas: High-resolution spectroscopy is actually a very sensitive, non-intrusive technique for detecting stable or unstable species and estimating their temperatures. Moreover, in this frequency range, spectra are usually not too congested, which makes the problem of identifying blended spectra less critical than in the infrared range.

Also, being inherently a very high resolution technique (typically of the order of $100 \mathrm{kHz}$ ), millimeter wave spectroscopy gives essential information about the energy-level configurations and molecular structure, making possible a detailed analysis of the rotational, fine and hyperfine structures, and leading to an accurate determination of the molecular parameters. The molecular structures determined by this method can be compared with the results of ab initio calculations, and provide reliable tests for the validity of the various levels of calculation. In this view, the reactive species are of special interest, since they widen the possibilities of comparing the bonding properties between atoms in the same column of the Mendeleieff table, such as carbon and silicon.

\section{Basic Principles}

In its principle, a millimeter wave spectroscopy experiment is extremely simple,as shown in Fig. 1, it is basically an absorption experiment. The source of radiation is a monochromatic, frequency-swept source, usually a klystron, a carcinotron, or, more recently, a gunn diode. The radiation passes through the absorption cell and a sensitive detector (generally a broadband $\mathrm{InSb}$ bolometer) is used to detect the absorption resulting from the interaction of the molecules with the electromagnetic field.

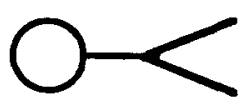

radiation source

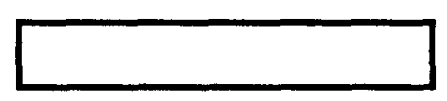

absorption ce11

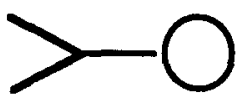

detector
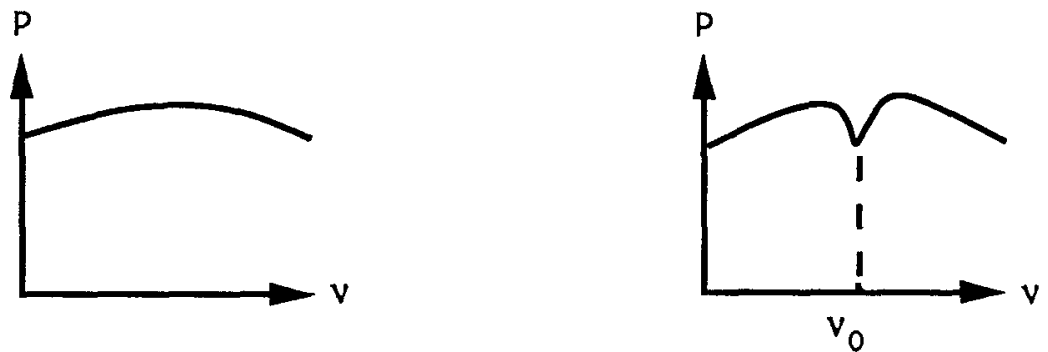

Fig. 1. Basic principle of an experiment of millimeter wave spectroscopy

Since the absorption of radiation by an atom or a molecule is mainly due to its electric dipole moment, only polar molecules can be detected by this method. 
A few non-polar molecules have been detected through magnetic dipole transitions, like $\mathrm{O}_{2}$ (Miller \& Townes 1953), but the corresponding absorption is usually weak, and this is a severe limitation in the application of radio astronomy to the detection of interstellar molecules.

In order to avoid collisional broadening of the transitions, the gas in the absorption cell must be kept at a rather low pressure, ranging typically from 1 to $10 \mathrm{P}$. In this pressure range, the linewidth is of the order of a few hundred $\mathrm{kHz}$, and millimeter wave spectroscopy is characterized by a high resolution (of the order of 50 to $100 \mathrm{kHz}$ ).

In the frequency range typical of millimeter wave spectroscopy (currently from 60 to $300 \mathrm{GHz}$ approximatively; up to 400 to $600 \mathrm{GHz}$ in more special cases), molecular transitions occur between rotational levels within a given vibrational state. Since the reactive species of interest are generally small molecules (4 to 5 atoms or less), their spectra are usually not very dense, and the probability of getting blended lines is rather small. This characteristic of millimeter wave spectroscopy is at the origin of the 2 different types of information which can be found in such a spectrum:

The first situation happens when enough lines have been measured and identified (which is not always straightforward!): the spectrum can be fitted using conventional models, and molecular constants are derived. The interpretation of these molecular constants gives information on the electronic and geometrical structure of the molecule. In particular, the rotational constants $\mathrm{A}, \mathrm{B}, \mathrm{C}$ are in first approximation inversely proportional to the inertial moments of the molecules, and, when several isotopic forms of a molecule have been observed, (i.e. several set of rotational constants corresponding to the same geometrical structure, but to different atomic masses), an accurate molecular structure can be determined. Of course, for a discussion of the accuracy on the bond lengths and angles, the validity of the theoretical model must be very carefully checked, but such a discussion is irrelevant in this paper. Moreover, knowing the molecular constants and using the convenient theoretical model, it is possible to calculate with an accuracy comparable to the experimental uncertainty the lines which, sometimes, cannot be directly measured for technical reasons.

The second situation happens when the spectrum of a molecule is already known: it is not necessary to observe the complete spectrum to identify this molecule in a complicated mixture: since the lines are rarely blended, the identification can rely on the observation of only a few characteristic lines, in contrast to infrared spectroscopy, where the identification is much more difficult. This property of millimeter wave spectra is of special importance for radio astronomy. 


\section{Experimental Problems}

Even if the principles of the experiment are very simple, its realization confronts the spectroscopists with many problems: First of all, the frequency range to be swept must be roughly defined, or, in other words, the line frequencies must be predicted. Then, the spectrometer must be very sensitive, easily scanned in frequency, and, as much as possible, automatized in order to save time. The last problem, but not the least, is to produce the reactive species which, in terrestrial conditions, are usually very short-lived.

\section{Prediction of the spectra}

An ideal case is of course when the line frequency is already known. This condition occurs when the problem is to confirm, by laboratory observation, the assignment of a line observed by radio astronomy, (as mentioned above in the case of $\mathrm{HCO}^{+}$). $\mathrm{HCO}^{+}$is not the only example, other ions, like $\mathrm{HCS}^{+}$(Thaddeus et al. 1981; Gudeman et al. 1981) and $\mathrm{HOCO}^{+}$(Thaddeus et al. 1981; Bogey et al. 1984) were also observed first by radio astronomy and then identified in the laboratory.

Important information can also be derived from other types of spectra, electronic spectra (see for example $\mathrm{SiC}_{2}$, Michalopoulos et al. 1984; Gottlieb et al. 1989) or vibrational spectra, as in the case of HNSi (Elhaninne et al. 1991; Bogey et al. 1991a) or $\mathrm{C}_{2} \mathrm{H}_{3}^{+}$(Bogey et al. 1992b). When no experimental data is available, the prediction of the spectra relies entirely on ab initio calculations. $\mathrm{Ab}$ initio calculations provide the potential energy surfaces, the minima of which correspond to the stable forms of the molecules. They also provide the values of the molecular parameters (bond lengths and angles) corresponding to the equilibrium structures of the molecules. From these parameters, the values of the rotational constants can be easily calculated. Due to the high resolution of millimeter wave spectroscopy, the accuracy of these ab initio calculations was for a long time not sufficient to allow an unambiguous identification of a molecule (see the example of $\mathrm{HCO}^{+}$, Klemperer 1970). Fortunately, the accuracy of ab initio calculations has improved very much during the last years, and, as shown later, the role of quantum chemists is sometimes crucial in the procedure of identification of molecular spectra (see for example Bogey et al. 1987; 1991b; 1992a; Cordonnier et al. 1992).

\section{The spectrometer}

Since free radicals and molecular ions are very reactive species, their stationary concentration is very low, and the main quality of the spectrometer is a sensitivity as high as possible. A conservative value for the sensitivity of a spectrometer working in the $50-450 \mathrm{GHz}$ range is $\Delta \mathrm{P} / \mathrm{P}=10^{-7}$ per $\mathrm{cm}$ of absorption path 
(provided a convenient radiation source is used). Using typical values for the various parameters (dipole moment, linewidth, oscillator strength...), it is possible to estimate the lowest detectable concentration to be $10^{8}$ to $10^{7}$ per $\mathrm{cm}^{3}$, which makes millimeter wave spectroscopy a competitive technique to detect reactive species, especially for the closed-shell molecules which are difficult to study by visible or UV spectroscopy.

The spectrometer we have built in Lille has been described elsewhere (Bogey et al. 1993). Below $340 \mathrm{GHz}$, we use harmonic generation from phase-locked klystrons or Gunn oscillators. Above $340 \mathrm{GHz}$, we use two phase-locked Thomson CSF carcinotrons emitting in the $340-470 \mathrm{GHz}$ frequency range. In this frequency range, the high power emitted by the radiation source allows a very good sensitivity to be reached (about $10^{-8} \mathrm{~cm}^{-1}$ for a $10 \mathrm{~Hz}$ bandwidth detection). The detection is achieved by a QMC Instrument liquid-helium-cooled InSb detector. The radiation is frequency-modulated, and the signal is demodulated at twice the modulation frequency, which provides a second derivative lineshape. A HP 9000-310 micro computer is used to automatically sweep the frequency and simultaneously record the spectrum. It also ensures frequency measurement and, when necessary to improve the signal-to-noise ratio, numerical signal processing.

\section{Methods of production}

Due to their short lifetime, reactive species can be detected only when they are produced directly in the absorption cell. Depending on the type of molecule of interest, various techniques can be used: Relatively fragile, moderately reactive molecules need soft chemical methods, like vacuum flash thermolysis and/or vacuum gas-solid phase reactions, which were used together to produce the $2 \mathrm{H}$ azirine $\mathrm{NCH}_{2} \mathrm{CH}$, a cyclic isomer of methyl cyanide (Bogey et al. 1986). In the case of very reactive free radicals and molecular ions, the species are most conveniently produced and observed within a plasma excited by a D.C. discharge, and we will restrict ourself to this case in this paper. In a D.C. discharge, two parts of the discharge are of special interest. Generally, the positive column is used when free radicals or reactive molecules are searched for. In normal conditions $(I=50-200 \mathrm{~mA})$, this part of the discharge fills nearly the whole length of the discharge tube. A very short part of the discharge, called the negative glow and located near the cathode, is more suitable for the production of molecular ions, and especially of protonated molecules, because of the higher electron density. In order to extend this negative glow to the whole length of the cell, an axial magnetic field (typically $200 \mathrm{G}$ ), produced by a solenoid coiled round the cell, is applied to the discharge (De Lucia et al. 1983). In this regime, the discharge is characterised by a low discharge current $(1-10 \mathrm{~mA})$. The magnetic field is also used to discriminate the lines due to an ion from those due to a neutral: The intensity of the lines due to ions is strongly correlated to the strength of the magnetic field, whereas those due to neutral are insensitive to the field. (De Lucia et al. 1983; Bogey et al. 1988). Furthermore, when the positive column is 
used, the magnetic field is also very useful to check the Zeeman effect on the lines, in order to discriminate paramagnetic species from others.

A scheme of the discharge tube built in Lille is presented in Fig. 2. The cell consists of a $2.5 \mathrm{~m}$ long, $5 \mathrm{~cm}$ internal diameter Pyrex tube, with a jacket for liquid nitrogen cooling. Condensable gases are introduced through a $2 \mathrm{~m}$ long axial glass tube drilled all along its length. Both ends are closed by Teflon windows at Brewster incidence to improve the transmission of the cell.

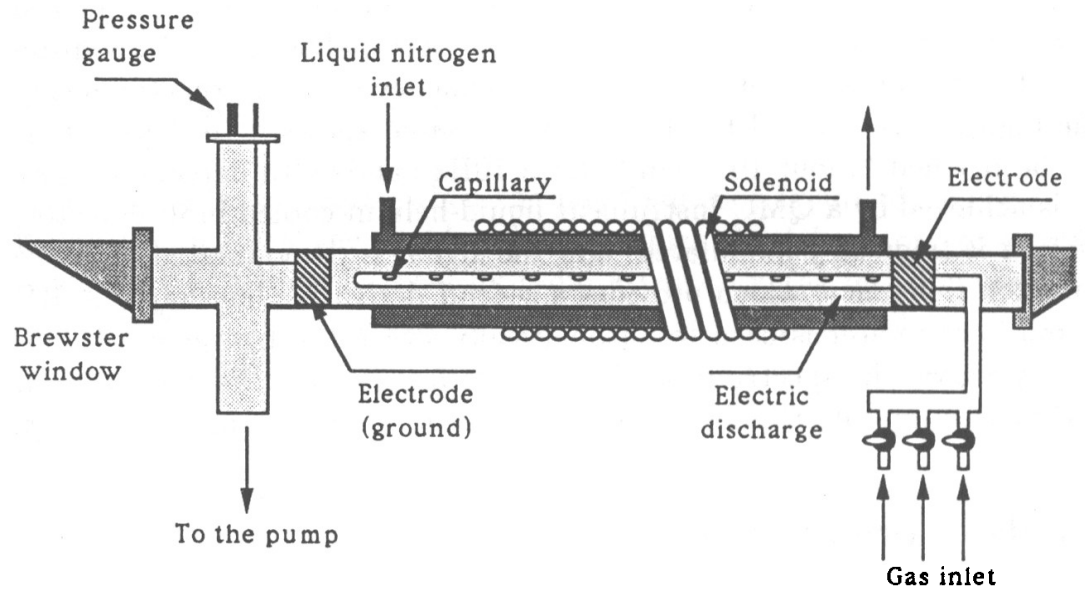

Fig. 2. Schematic diagram of the discharge cell

\section{Experimental Procedure}

To achieve a detection, measurement, and identification of the millimeter wave spectrum of a reactive species, not only good frequency predictions, a very sensitive spectrometer and an efficient technique of production are necessary: A bit of good luck is also essential, as well as lots of patience.

The main problem is that the physico-chemistry of the electric discharge is extremely mysterious, and in many cases our understanding of what happens in the discharge is nearer cooking recipe than conventional scientific argument. In these conditions, the only procedure is to scan the convenient spectral range (when correctly estimated) in various experimental conditions: chemical mixture, total and partial pressures, temperature and discharge conditions. Of course, sometimes it is successful, sometimes it is not. But the most fascinating is that, in some case, during an unsuccessful search for a given molecule, we found another species, much more interesting than the one we searched for first. In such a case, first the procedure of identification of the spectrum, and then of the molecule itself, and then possibly of its structure, is something like a detective story, as 
we experienced it first with the weakly bound molecular ion $\mathrm{ArH}_{3}+$ (Bogey et al. 1987), and will be described in the next section on other examples.

\section{Some Typical Examples}

The dibridged disilyne $\mathrm{Si}\left(\mathrm{H}_{2}\right) \mathrm{Si}$ : In the course of a search for the molecular ion $\mathrm{SiH}^{+}$, unidentified lines were observed in a silane/argon plasma, in the $450 \mathrm{GHz}$ frequency range. The lines had a typical behaviour, being maximum in intensity in the abnormal regime of the discharge (very low discharge current), and disappearing nearly completely in the positive column. However their intensity did not show the variation versus the magnetic field strength typical of "ionic" lines. Moreover, they did not exhibit any Zeeman splitting. After a few more chemical tests, we concluded that they were due to a closed-shell, neutral molecule containing only silicon and hydrogen atoms.

Figure 3 presents a stick diagram of a part of the observed spectrum. It shows the very characteristic pattern of a c-type $Q$ branch, and the assignment of this spectrum by conventional methods (Winnewisser et al. 1968) was straightforward. Finally, a total of 87 lines was measured in the $347-472 \mathrm{GHz}$ range, and the spectrum was fitted using a conventional Hamiltonian, with a standard deviation of $20 \mathrm{kHz}$.

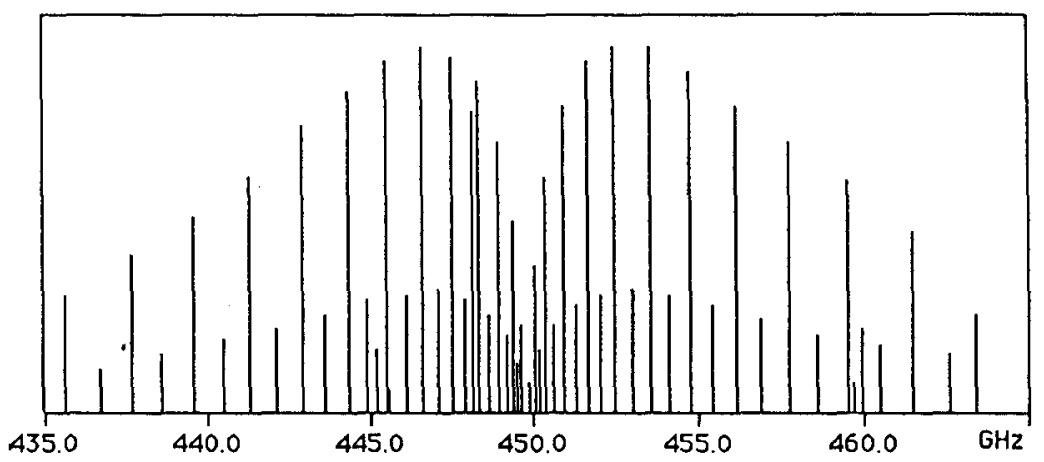

Fig. 3. Stick diagram of a part of the observed spectrum

The identification of this unknown molecule relied on a careful examination of the values of the rotational constants $\mathrm{A}, \mathrm{B}, \mathrm{C}$, and of the characteristics of the observed spectrum:

(a) The $\mathrm{B}$ and $\mathrm{C}$ constants $(=7250 \mathrm{MHz})$ are of the same order of magnitude as those of molecules like $\mathrm{S}_{2}(\mathrm{~B}=8.8 \mathrm{GHz})$ or $\mathrm{Si}_{2}(\mathrm{~B}=7.2 \mathrm{GHz})$, which indicates that the molecule contains 2 silicon atoms. 
(b) The relatively high value of $\mathrm{A}(=157 \mathrm{GHz})$ suggests that $\mathrm{A}$ is mainly determined by a few $\mathrm{H}$ atorns out of the $\mathrm{Si}-\mathrm{Si}$ axis, and that the number of $\mathrm{H}$ atoms do not exceed 3 (by comparison with known molecules).

(c) The remarkable 1:3 alternation in the intensities of adjacent lines, shown on Fig. 3, is characteristic of 2 exchangeable hydrogen atoms. All these observations suggests that the observed molecule is the disilyne $\mathrm{Si}_{2} \mathrm{H}_{2}$, a fundamental molecule never observed spectroscopically, but theoretically studied by quantum chemistry methods (Colegrove \& Schaefer 1990; Grev \& Schaefer 1992).

(d) These ab initio calculations showed that the ground state of $\mathrm{Si}_{2} \mathrm{H}_{2}$ has a singlet non-classical bridged structure (Fig. 4). With such a $\mathrm{C}_{2 v}$ symmetry, the molecule is polar, and, with the calculated structure, the dipole moment lies along the c-axis, in agreement with the experiment.

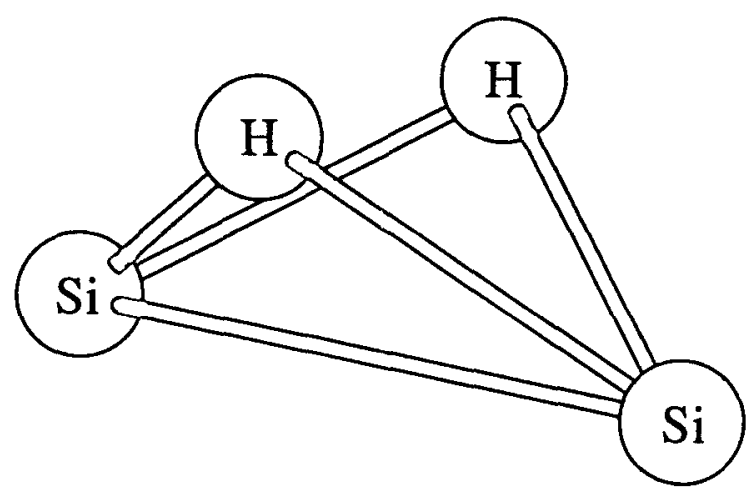

Fig. 4. Molecular structure of the disilyne $\mathrm{Si}\left(\mathrm{H}_{2}\right) \mathrm{Si}$

(e) The A,B,C constants calculated from the ab initio structure are close to the experimental values, as shown on Table 1.

(f) Other potential molecules can be ruled out in view of spectroscopic arguments, for example: the silasilene polar isomer $\mathrm{H}_{2} \mathrm{SiSi}$ would give rise to an a-type spectrum, very different from the observed spectrum. $\mathrm{Si}_{2} \mathrm{H}_{4}$ has a transbent geometry in its ground state, with a center of symmetry, and then no dipole moment.

A definite confirmation of the identification of the molecule came with the observation of the ${ }^{29} \mathrm{Si}$ and ${ }^{30} \mathrm{Si}$ monosubstituted forms: The rotational constants were calculated assuming the structure derived from ab initio calculations, and the spectra were predicted using a conventional hamiltonian. The spectra were observed in natural abundance, and a set of rotational constants was derived for each isomer (Bogey et al. 1991b). Later, the $\mathrm{Si}\left(\mathrm{D}_{2}\right) \mathrm{Si}$ spectrum was also observed 
Table 1. Rotational constants of $\mathrm{Si}\left(\mathrm{H}_{2}\right) \mathrm{Si}$ (in $\mathrm{MHz}$ ). Calculations from Colegrove \& Schaefer (1990)

\begin{tabular}{crr}
\hline & Experiment & Calculation \\
\hline $\mathrm{A}$ & 157198.8 & 163450.0 \\
$\mathrm{~B}$ & 7281.3 & 7236.0 \\
$\mathrm{C}$ & 7199.7 & 7154.0 \\
$\mathrm{~B}-\mathrm{C}$ & 81.6 & 81.9 \\
\hline
\end{tabular}

by replacing $\mathrm{SiH}_{4}$ with $\mathrm{SiD}_{4}$ in the discharge cell, and another set of rotational constants was derived.

Due to the symmetry of the molecule, its structure depends only on 3 geometrical parameters, namely the $\mathrm{Si}-\mathrm{H}$ and $\mathrm{Si}-\mathrm{Si}$ distances, and the dihedral angle $<\mathrm{HSiSiH}>$. Using together the 4 sets of rotational constants, it was possible to derive a substitution structure (Kraitchman 1953; Costain 1958) which is a very good approximation of the equilibrium structure. As shown in Table 2, the agreement between experimental and theoretical structures is excellent (Bogey et al. 1993).

Table 2. Molecular structure of $\mathrm{Si}\left(\mathrm{H}_{2}\right) \mathrm{Si}$

\begin{tabular}{crrr}
\hline & This work & ref(1) & ref $(2)$ \\
\hline $\mathrm{Si}-\mathrm{Si}(\AA)$ & 2.2154 & 2.216 & 2.222 \\
$\mathrm{Si}-\mathrm{H}(\AA)$ & 1.6680 & 1.668 & 1.681 \\
$\left\langle\mathrm{H} \mathrm{SiSi} \mathrm{H}>\left(^{\circ}\right)\right.$ & 104.22 & 104.0 & 106.5 \\
\hline
\end{tabular}

(1) Colegrove \& Schaefer 1990, (2) Grev \& Schaefer 1992.

\section{The Monobridged Form $\mathrm{Si}(\mathrm{H}) \mathrm{SiH}$}

Another case of very interesting unidentified lines occurred during a search for $\mathrm{SiH}_{3}$. This radical is clearly of astrophysical interest, but there is a large uncertainty on its spectrum because it presents both spin-orbit coupling and inversion internal motion. Several frequency scans were carried out in various experimental conditions: discharge in pure silane or in argon-silane mixtures, at room temperature or with liquid nitrogen cooling of the cell. Again, a lot of unidentified lines were observed, which cannot be ascribed to $\mathrm{SiH}_{3}$. Clearly, the molecule responsible for these lines was a short-lived molecule, appearing only in the abnormal regime of the discharge, it was not an ion, and it was a closed-shell molecule. Moreover, this mysterious molecule had a chemical behaviour very similar to that of the dibridged disilyne $\mathrm{Si}\left(\mathrm{H}_{2}\right) \mathrm{Si}$. From a spectroscopic point of view , a $6 \mathrm{GHz}$ 
frequency scan around $210 \mathrm{GHz}$ allowed the observation of a typical a-type pattern, with no indication of spin statistics from the relative intensities of the lines.

The identification of the K-components was straightforward, but several trials were necessary to determine the J-value. Finally, the spectrum was identified and fitted, and line predictions were made in the $420-470 \mathrm{GHz}$ frequency range. It turned out that these predictions allowed us to identify some unassigned lines observed during our preceeding study of $\mathrm{Si}\left(\mathrm{H}_{2}\right) \mathrm{Si}$. A total of 160 lines were finally measured between 180 and $470 \mathrm{GHz}$. They were fitted using a conventional hamiltonian in order to derive the molecular constants. As shown in Table 3 , there is a striking similarity between the $\mathrm{B}$ and $\mathrm{C}$ constants of $\mathrm{Si}\left(\mathrm{H}_{2}\right) \mathrm{Si}$ and the unknown molecule. It means that, here again, the molecule contains only 2 silicon atoms.

Table 3. Rotational constants (in $\mathrm{MHz}$ ) of the unknown molecule and $\mathrm{Si}\left(\mathrm{H}_{2}\right) \mathrm{Si}$

\begin{tabular}{rrr}
\hline & $\begin{array}{c}\text { unknown } \\
\text { molecule }\end{array}$ & \multicolumn{1}{c}{$\mathrm{Si}\left(\mathrm{H}_{2}\right) \mathrm{Si}$} \\
\hline $\mathrm{A}$ & 262092.3 & 157198.8 \\
$\mathrm{~B}$ & 7361.9 & 7281.3 \\
$\mathrm{C}$ & 7161.8 & 7199.7 \\
\hline
\end{tabular}

Here again, the identification of the molecule was greatly aided by using the results of ab initio calculations. Indeed, in the papers where the results concerning $\mathrm{Si}\left(\mathrm{H}_{2}\right)$ Si were presented (Colegrove \& Schaefer 1990; Grev \& Schaefer 1992), the authors also characterised the other stationary points on the $\mathrm{Si}_{2} \mathrm{H}_{2}$ potential surface. In addition to the already known disilavinylidene isomer $\mathrm{SiSiH}_{2}$ (see for example Koseki \& Gordon 1989), they discovered another low-lying isomer, the monobridged form $\mathrm{Si}(\mathrm{H}) \mathrm{SiH}$, which turned out in fact to be lower in energy than the disilavinylidene. They also showed that the energy difference between the di- and mono-bridged forms is low enough $(8.7 \mathrm{kcal} / \mathrm{mol})$ to make this isomer an attractive candidate for our unknown molecule. Not only were the calculated rotational constants of this new isomer in good agreement with the experimental values (see Table 4), but other arguments were much more in favour of $\mathrm{Si}(\mathrm{H}) \mathrm{SiH}$ than of $\mathrm{SiSiH}_{2}$ too. $\mathrm{SiSiH}_{2}$ has 2 exchangeable $\mathrm{H}$ atoms, which is not consistent with our observations. Moreover, it has a rather small a-type dipole moment $(0.15 \mathrm{D})$. In contrast to this, $\mathrm{Si}(\mathrm{H}) \mathrm{SiH}$ has no equivalent $\mathbf{H}$ atoms, its dipole moment has 2 components, the strongest $(0.96 \mathrm{D})$ along the a-axis, and a much weaker one (0.04D) along the b-axis, which is consistent with the typical a-type spectrum observed.

As in the preceeding case, the definite confirmation of the identification of the molecule requested the observation of another isotopomer. The spectra of the ${ }^{29} \mathrm{Si}$ and ${ }^{30} \mathrm{Si}$ monosubstituted forms were too weak to be observed in natural abundance, but the deuterated form was observed by replacing $\mathrm{SiH}_{4}$ by $\mathrm{SiD}_{4}$ in 
Table 4. Rotational constants (in $\mathrm{MHz}$ ) of the unknown molecule and the $\mathrm{Si}_{2} \mathrm{H}_{2}$ isomers (Calculations from Grev \& Schaefer 1992)

\begin{tabular}{lclc}
\hline & A & B & C \\
\hline Experimental & 262092.3 & 7361.9 & 7161.8 \\
SiSiH $_{2}$ & 168663.0 & 6708.0 & 6452.0 \\
$\mathrm{Si}(\mathrm{H}) \mathrm{SiH}$ & 275510.0 & 7375.0 & 7181.0 \\
\hline
\end{tabular}

the discharge. As expected, the spectrum was observed very near the predicted spectrum, and the observed spectrum was fitted to derive the molecular constants. In the case of this molecule, 5 geometrical parameters are necessary to determine the structure, for example the $\mathrm{Si}-\mathrm{Si}$ distance, $2 \mathrm{Si}-\mathrm{H}$ distances, and 2 angles (see Fig. 5). With 2 isomers, only 4 independent rotational constants are available, since, for a planar molecule, the 3 rotational constants are not independent. It is then impossible to determine completely the molecular structure from the experimental data. In order to determine a preliminary structure, the $\left.<\mathrm{Si}_{1}-\mathrm{Si}_{2}-\mathrm{H}_{1}\right\rangle$ angle was arbitrarily fixed to the ab initio value of $52.5^{\circ}$. It led to the following values for the various structural parameters: $\mathrm{Si}_{1}-\mathrm{Si}_{2}=2.119 \AA$; $\left.\mathrm{Si}_{2}-\mathrm{H}_{1}=1.629 \AA ; \mathrm{Si}_{2}-\mathrm{H}_{2}=1.474 \AA ;<\mathrm{Si}_{1}-\mathrm{Si}_{2}-\mathrm{H}_{2}\right\rangle=157.5 \circ$ (Cordonnier et al. 1992).

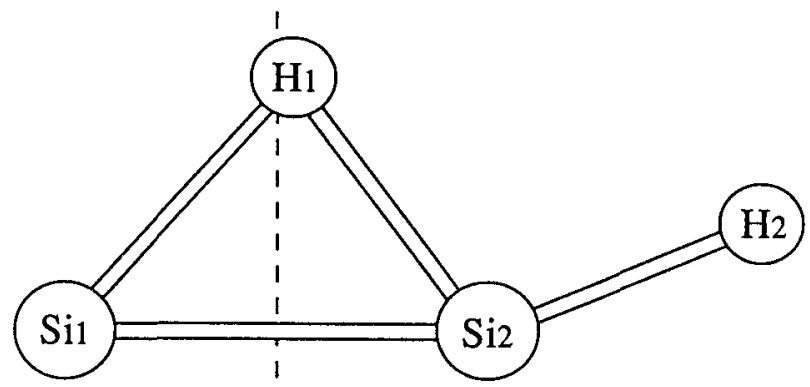

Fig. 5. Molecular structure of the monobridged $\mathrm{Si}(\mathrm{H})) \mathrm{SiH}$

\section{The Protonated Acetylene $\mathrm{C}_{2} \mathrm{H}_{3}{ }^{+}$}

The observation of protonated species was early suggested as an indirect way to detect non polar molecules like acetylene, $\mathrm{C}_{2} \mathrm{H}_{2}$, in the interstellar medium (Herbst et al. 1977). Moreover, protonated acetylene $\mathrm{C}_{2} \mathrm{H}_{3}{ }^{+}$is expected to play an important role in the hydrocarbon chemistry of the interstellar medium (Herbst \& Leung 1989), and Glassgold et al. (1992), reexamined the chemistry of this ion, and concluded that with the existing large radiotelescopes some millimeter wave transitions should be observable in interstellar clouds and/or C-rich circumstellar envelopes. 
$\mathrm{C}_{2} \mathrm{H}_{3}^{+}$has also received considerable attention from the quantum chemists, due to the possible existence of classical and non-classical (bridged) structures in this small carbo-cation (Fig. 6). From the various ab initio calculations, it seems to emerge that the classical structure is a transition state between two equivalent forms of the non-classical isomer (Lindh et al. 1991; see also Crofton et al. 1989 for a review of earlier works). The height of the barrier is estimated to be about $1300 \mathrm{~cm}^{-1}$ (Lindh et al. 1991), which is low enough to allow tunneling on a measurable time scale.

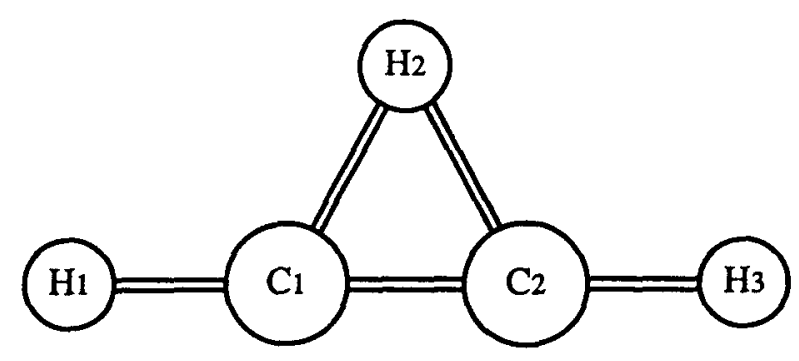

Non-classical form

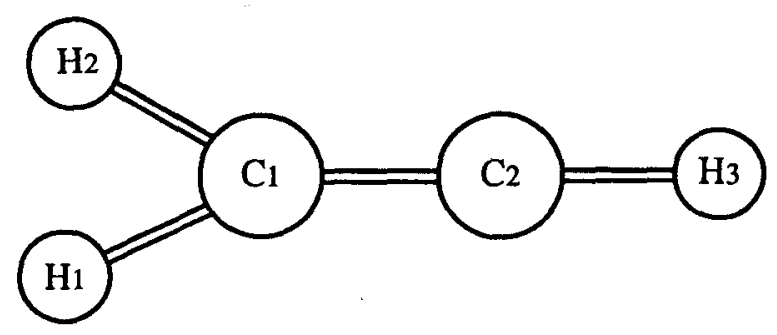

Classical form

Fig. 6. Molecular structures of the protonated acetylene $\mathrm{C}_{2} \mathrm{H}_{3}^{+}$

In contrast to these numerous theoretical results, very little was known experimentally on the spectroscopic properties of this ion, until the pioneering work of Oka's group in Chicago. They first observed infrared lines of $\mathrm{C}_{2} \mathrm{H}_{3}+$ in 1985 (Crofton \& Oka 1985), and published recently their main conclusions (Crofton et al. 1989): The very rich infrared spectrum observed in the $3.2 \mu \mathrm{m}$ range is due to the bridged form of $\mathrm{C}_{2} \mathrm{H}_{3}{ }^{+}$. Moreover, tunneling splittings expected from the moderate barrier height were experimentally resolved in the excited vibrational state. However, in spite of extensive theoretical works (Hougen 1987; Escribano\& Bunker 1987; Escribano et al. 1988; Gomez \& Bunker 1990), there is presently 
no theoretical model to interpret quantitatively this tunneling splitting within experimental accuracy. Consequently, Crofton et al. (1989) used infrared line combination differences to determine the $\mathrm{B}$ and $\mathrm{C}$ rotational constants of $\mathrm{C}_{2} \mathrm{H}_{3}^{+}$ in its ground vibrational state, but were unable to determine $A$, preventing any accurate prediction of the pure rotational b-type spectrum to be made.

However, we decided to carry out a search for this rotational spectrum, since, in view of the reasonably large dipole moment $(\simeq 1.25 \mathrm{D}$ according to Lee \& Schaefer 1986), it could be observed at $\mathrm{cm}$ and $\mathrm{mm}$ wavelengths (Glassgold et al. 1992).

The ions were produced in the negative glow discharge, in a mixture of argon, hydrogen and acetylene. A $5 \mathrm{GHz}$ range was scanned around $432 \mathrm{GHz}$, the frequency of the intense $9_{18}-9_{09}$ transition predicted using the molecular constants of Crofton et al. (1989), and several lines were observed. Among them, only one exhibited the intensity evolution versus magnetic field characteristic of an ion. Several chemical tests were performed to make sure that the ion contains only $\mathrm{C}$ and $\mathrm{H}$ atoms. Assuming that this line was indeed the expected transition, its frequency was included in a fit together with the combination differences of Crofton et al. (1989), leading to a preliminary determination of the rotational constant $A$, and to a prediction of the frequencies of other transitions, which were readily observed.

A total of 17 b-type transitions were finally measured and fitted using a standard hamiltonian, leading to the determination of the 3 rotational constants and 4 centrifugal distortion constants (Bogey et al. 1992b). In order to confirm the identification of the molecule, we compared the molecular constants obtained by fitting (a) only the combination differences of Crofton et al. (1989); (b) both the combination differences and the pure rotational transitions; (c) only the rotational transitions. The results are presented in Table 5. Their consistency is remarkable and confirms that the molecular ion observed by Oka's group and by us is the same carbo-ion, namely $\mathrm{C}_{2} \mathrm{H}_{3}{ }^{+}$in its non-classical, bridged form. Moreover, all ab initio calculated rotational constants are in agreement with these results.

Table 5. Rotational constants of $\mathrm{C}_{2} \mathrm{H}_{3}+$ (in $\mathrm{MHz}$ )

\begin{tabular}{rrrr}
\hline & IR data & IR + mmw data & mmw data \\
\hline $\mathrm{A}$ & $396925 .($ fixed) & $399956.00(17)$ & $399955.983(18)$ \\
$\mathrm{B}$ & $34234.8(20)$ & $34237.532(58)$ & $34237.5395(66)$ \\
$\mathrm{C}$ & $31371.8(20)$ & $31371.74(12)$ & $31371.760(14)$ \\
\hline
\end{tabular}

One of the most exciting problems concerning this molecule is related to the internal motion of the protons. In spite of an extensive search, no splitting of the 
lines resulting from this tunneling motion was observed in the pure rotational spectrum. This result indicates that the barrier is probably higher than the calculated value (Lindh et al. 1991), and this problem should be re-investigated in the future, since the tunneling splitting is expected to be very sensitive to the height of the barrier (Escribano 1993).

Finally, the frequencies of some (for technical reasons) non observed lines of potential astrophysical interest were predicted using our set of molecular constants (Bogey et al. 1992b).

Recently, this molecular ion was searched for in the interstellar medium. The detection was negative, but an upper limit for its abundance was determined (Guelin 1993).

\section{Conclusion}

Recent improvements in the methods of production as well as in the performances of the spectrometers (sensitivity, frequency coverage, automatisation) have made possible the spectacular developments in the topic of high resolution spectroscopy of transient species. From the examples presented above, it is clear that quantum chemistry plays often a key-role in the procedure of identification of a new molecule.

The necessary interplay between theory, laboratory spectroscopy and radio astronomy has proved to be particularly successful, and many exciting discoveries are still expected in this field, as examplified by the recent laboratory and radio astronomical detection of $\mathrm{MgNC}$, the first magnesium-containing interstellar molecule (Kawaguchi et al. 1993). It is also noteworth that radio astronomy is not only a strong motivation for laboratory spectroscopists, it can be also, indirectly, at the origin of the accidental discovery of fascinating molecules in the laboratory, as illustrated by $\mathrm{ArH}_{3}+$ (Bogey et al. 1988), $\mathrm{Si}\left(\mathrm{H}_{2}\right) \mathrm{Si}$ (Bogey et al. 1991b), and $\mathrm{Si}(\mathrm{H}) \mathrm{SiH}$ (Cordonnier et al. 1992).

Acknowledgement. This research was partially supported by the European Communities (project 892001 59/OP1: "Structure and Dynamics of Molecular Ions") and by the C.N.R.S. (Groupe De Recherche "Physico-Chimie des Molecules et Grains Interstellaires").

\section{References}

Bogey M., Demuynck C., Destombes J.L., 1984, Astron. Astrophys., 138, L11

Bogey M., Destombes J.L., Denis J.M., Guillemin J.C., 1986, J. Mol. Spectr., 115 , 1 Bogey M., Bolvin H., Demuynck C., Destombes J.L., 1987, Phys. Rev. Lett., 58, 988 
Bogey M., Demuynck C., Destombes J.L., Walters A., 1991a, Astron. Astrophys., 244, $\mathrm{L} 47$

Bogey M., Bolvin H., Demuynck C., Destombes J.L., 1991b, Phys. Rev. Lett., 66, 413

Bogey M., Cordonnier M., Demuynck C., Destombes J.L., 1992a,J. Mol. Spectr., 155, 217

Bogey M., Cordonnier M., Demuynck C., Destombes J.L., 1992b, Astrophys. J., 399, L103

Bogey M., Bolvin H., Cordonnier M., Demuynck C., Destombes J.L., Csaszar A.G., 1993 , in preparation

Buhl D., Snyder L.E., 1970, Nature, 228, 267

Colegrove B.T., Schaefer III H.F., 1990, J. Phys. Chem., 94, 5593

Cordonnier M., Bogey M., Demuynck C., Destombes J.L., 1992, J. Chem. Phys., 97, 7984

Costain C.C., 1958, J. Chem. Phys., 29, 864

Crofton M.W., Oka T., 1985, 40th Symposium on Molecular Spectroscopy, Columbus, Ohio

Crofton M.W., Jagod M.F., Rehfuss B.D., Oka T., 1989, J. Chem. Phys., 91, 5139

De Lucia F.C., Herbst E., Plummer G.M., Blake G.A., 1983, J. Chem. Phys., 78, 2312

Destombes J.L., Demuynck C., Bogey M., 1988, Phil. Trans. R. Soc. Lond. A, 324, 147

Dousmanis G.C., Sanders T.M. Jr, Townes C.H., 1955, Phys. Rev., 100, 1735

Elhanine M., Farrenq R., Guelachvili G., 1991, J. Chem. Phys., 94, 2529

Escribano R., Bunker P.R., 1987, J. Mol. Spectr., 122, 325

Escribano R., Bunker P.R., Gomez P.C.,1988, Chem. Phys. Lett., 150, 60

Escribano R., 1993, private communication

Glassgold A.E., Omont A., Guelin M., 1992, Astrophys. J., 396, 115

Gomez P.C., Bunker P.R., 1990, Chem. Phys. Lett., 165, 351

Gottlieb C.A., Vrtilek J.M., Thaddeus P., 1989, Astrophys. J., 343, L29

Grev R.S., Schaefer III H.F., 1992, J. Chem. Phys., 97, 7990

Gudeman C.S., Haese N.N., Piltch N.D., Woods R.C., 1981, Astrophys. J., 246, L47

Guelin M., 1992, private communication

Herbst E., Green S., Thaddeus P., Klemperer W., 1977, Astrophys. J., 215, 503

Herbst E., Leung C.M., 1989, Astrophys. J. Suppl. Ser., 69, 271

Hougen J.T., 1987, J. Mol. Spectr., 123, 197

Kawaguchi K., Kagi E., Hirano T., Takano S., Saito S., 1993, Astrophys. J., 406, L39

Klemperer W., 1970, Nature, 227, 267

Koseki S., Gordon M.S., 1989, J. Phys. Chem., 93, 118

Kraemers W.P., Diercksen G.H.F., 1976, Astrophys. J. , 205, L97

Kraitchmann J., 1953, Am. J. Phys., 21, 17

Lee T.J., Schaefer III H.F., 1986, J. Chem. Phys., 85, 3437

Lindh R., Rice J.E., Lee T.J., 1991, J. Chem. Phys., 94,8008

Michapopoulos D.L., Geusic M.E., Langridge-Smith P.R.R., Smalley R.E., 1984, J. Chem. Phys., 80, 3556

Miller S.L., Townes C.H., 1953, Phys. Rev., 90, 537

Snyder L.E., Hillis J.M., Lovas F.J., Ulich B.L., 196, Astrophys. J., 209, 67

Thaddeus P., Guelin M., Linke R.A., 1981, Astrophys. J., 246, L41

Viala Y.P., 1986, Astron. Astrophys. Suppl. Ser., 64, 391

Wahlgreen U., Liu B., Pearson P.K., Schaefer III H.F., 1973, Nature, 246, 4

Winnewisser G., Winnewisser M., Gordy W., 1968, J. Chem. Phys., 49, 3465

Woods R.C., Dixon T.A., Saykally R.J., Szanto P.G., 1975, Phys. Rev. Lett., 35, 1269 